\title{
Excitation and deexcitation processes in atom-Rydberg atom collisions in helium-rich white dwarf atmospheres (Research Note)
}

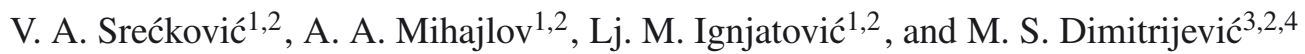 \\ ${ }^{1}$ University of Belgrade, Institute of physics, PO Box 57, 11001 Belgrade, Serbia \\ e-mail: vlada@ipb.ac.rs \\ 2 Institute Isaac Newton of Chile, Yugoslavia Branch, 11060 Belgrade, Serbia \\ 3 Astronomical Observatory, Volgina 7, 11060 Belgrade 74, Serbia \\ ${ }^{4}$ Observatoire de Paris, 92195 Meudon Cedex, France
}

Received 6 November 2012 / Accepted 14 February 2013

\section{ABSTRACT}

\begin{abstract}
We aim to show the importance of non-elastic excitation and deexcitation processes in $\mathrm{He}^{*}(n)+\mathrm{He}\left(1 \mathrm{~s}^{2}\right)$ collisions with the principal quantum number $n \geq 3$ for helium-rich white dwarf atmospheres. We compare the efficiencies of these processes with those of the known non-elastic electron- $\mathrm{He}^{*}(n)$ atom processes in the atmospheres of some DB white dwarfs. We show that in significant parts of the considered atmospheres, which contain weakly ionized layers (the ionization degree $\lesssim 10^{-3}$ ), the influence of the studied atomRydberg atom processes on excited helium atom populations is dominant or at least comparable to the influence of the concurrent electron-He ${ }^{*}(n)$-atom processes.
\end{abstract}

Key words. atomic processes - astrochemistry - stars: atmospheres - white dwarfs

\section{Introduction}

We here continue our previous investigations of inelastic $A+$ $A^{*}(n)$ atom-Rydberg atom collision processes (where the principal quantum number was $n \gg 1$ and $A$ was the ground-state atom), which influence the excited atom populations in stellar atmospheres. We consider the processes that can be interpreted as resulting from a resonant energy exchange between the subsystem $A+A^{+}$, in which $A^{+}$is the core of the Rydberg atom $A^{*}(n)$, and the outer electron of this atom. There are two groups of such processes. The first group involves the chemiionization processes (Janev \& Mihajlov 1980; Mihajlov et al. 1996, 1997), which connect the block of atomic Rydberg states with the continuum. The second group comprises the so-called $\left(n-n^{\prime}\right)$-mixing processes within the above mentioned block of atomic Rydberg states (Mihajlov 1982; Mihajlov et al. 2004, 2008 ), i.e. the excitation and deexcitation processes that imply transitions between the Rydberg states with the principal quantum numbers $n$ and $n^{\prime} \neq n$.

The chemi-ionization processes in $A^{*}(n)+A$ collisions, together with their inverse processes, have been studied for both $A=\mathrm{H}$ and $A=\mathrm{He}$ cases, which are especially important for stellar atmospheres. Mihajlov et al. $(2003,2007)$ studied the influence of these processes on the excited hydrogen atom populations and the spectral line profiles in the atmosphere of an $\mathrm{M}$ red dwarf $\left(T_{\text {eff }}=3800 \mathrm{~K}\right)$ using the PHOENIX code. Mihajlov et al. $(1997,2011,2003)$ studied the potential influence of these processes on the excited hydrogen and helium atom populations in the atmospheres of Sun and of some DB white dwarfs.

However, the possible influence of the $\left(n-n^{\prime}\right)$-mixing processes in $A^{*}(n)+A$ collisions on the excited-atom populations in stellar atmospheres has only been studied for the $A=\mathrm{H}$ case in the context of the atmosphere of the Sun (Mihajlov et al. 2005). In the above mentioned studies concerning the solar atmosphere, the necessary calculations were performed within the corresponding non-local thermodynamic equilibrium (non-LTE) model proposed by Vernazza et al. (1981). The findings of these studies have shown that the efficiency of these processes in a major part of the solar photosphere is dominant with respect to the efficiency of the relevant concurrent excitation and deexcitation processes for $n=4,5$ and 6 , and is comparable with them for $n=7$ and 8 . The above mentioned studies concluded that the excitation and deexcitation processes in $\mathrm{H}^{*}(n)+\mathrm{H}$ collisions should be included ab initio into future non-LTE models of the solar atmosphere. The importance and effects of the atomRydberg atom $\left(n-n^{\prime}\right)$-mixing processes have also been analyzed in Barklem (2007) and Mashonkina (2009).

We found no non-LTE models similar to that of Vernazza et al. (1981) for the Sun that would have provided all data necessary for calculations as in Mihajlov et al. (2005), in relation to the atmospheres of DB white dwarfs and other similar helium-rich stellar atmospheres. However, since the influence of strong electromagnetic emission is the same in helium-rich atmospheres as in the Sun, the distribution shape of the helium excited-atom population probably deviates considerably from the Boltzmann distribution. Taking this into account and because the data on the relative efficiencies of atom-Rydberg atom processes and the relevant concurrent processes would be useful for future non-LTE stellar-atmosphere models, the chemi-ionization processes in $\mathrm{He}^{*}(n)+\mathrm{He}$ collisions were studied in Mihajlov et al. (2003) in the context of DB white dwarf atmospheres, within the models proposed by Koester (1980). In the current 
study, for the same reason and using the same models, the efficiencies of the excitation processes

$\mathrm{He}^{*}(n)+\mathrm{He}=\left\{\begin{array}{l}\operatorname{He}^{*}\left(n^{\prime}=n+p\right)+\mathrm{He}, \\ \mathrm{He}+\operatorname{He}^{*}\left(n^{\prime}=n+p\right),\end{array} \quad p \geq 1\right.$,

and the inverse deexcitation processes

$\mathrm{He}^{*}(n)+\mathrm{He}=\left\{\begin{array}{l}\operatorname{He}^{*}\left(n^{\prime}=n-p\right)+\mathrm{He}, \\ \mathrm{He}+\operatorname{He}^{*}\left(n^{\prime}=n-p\right),\end{array} \quad 0<p \leq n-3\right.$,

(where $n+p$ and $n-p$ are the principal quantum numbers of the final Rydberg states) are compared for $3 \leq n \leq 8$ with the efficiencies of the relevant concurrent processes. Here, we consider the well-known electron-excited-atom collision excitation/deexcitation processes

$\mathrm{He}^{*}(n)+\vec{e}=\mathrm{He}^{*}(n \pm p)+\overrightarrow{e^{\prime}}$,

where $\vec{e}$ and $\vec{e}^{\prime}$ denote a free electron in the initial and final states, respectively. The necessary calculations were performed using the models in Koester (1980) for DB white dwarf atmospheres with $\log g=8$ and $T_{\text {eff }}=12000 \mathrm{~K}$ and $T_{\text {eff }}=14000 \mathrm{~K}$.

\section{Theoretical remarks}

As several of the above mentioned studies have reported, the same dipole resonant mechanism (DRM) causes the non-elastic $\left(n-n^{\prime}\right)$-mixing and chemi-ionization processes in $A^{*}(n)+A$ collisions. The DRM was also discussed in detail in Mihajlov et al. (2012). For the $\left(n-n^{\prime}\right)$-mixing processes (1) and (2), with $A=\mathrm{He}$, the DRM assumes that they occur in the region $R_{n ; n \pm p} \ll r_{n}$, where $R$ is the internuclear distance and $r_{n} \sim n^{2}$ is the characteristic radius of atom $\mathrm{He}^{*}(n)$, in the vicinity of the corresponding resonant points $R_{n ; n \pm p}$. These parameters are found to be the roots of the equation $U_{2}(R)-U_{1}(R)=\varepsilon_{n \pm p}-\varepsilon_{n}$, where $\varepsilon_{n}$ and $\varepsilon_{n \pm p}$ denote the energies of the Rydberg states $\mid n>$ and $\mid n \pm p>$ with the principal quantum numbers $n$ and $n \pm p$; and $U_{1}(R)$ and $U_{2}(R)$ are the adiabatic energies of the electronic ground state $\mid 1 ; R>$ and the first excited state $\mid 2 ; R>$ of the molecular ion $\mathrm{He}_{2}^{+}$. We took the potential curves $U_{1}(R)$ and $U_{2}(R)$ from Ignjatović et al. (2009) and following the foundational work of Janev \& Mihajlov (1979), which focuses on the $\left(n-n^{\prime}\right)$-mixing processes, we assumed that the processes (1) and (2) are caused exclusively by the transitions $|n>\rightarrow| n+p>$ and $|n>\rightarrow| n-p>$ of the outer electron, which occur simultaneously with the transitions $|2 ; R>\rightarrow| 1 ; R>$ and $|1 ; R>\rightarrow| 2 ; R>$, respectively, in the subsystem $\mathrm{He}^{+}+\mathrm{He}$, close to the resonant points $R_{n ; n \pm p}$. This fact is responsible for the resonant character of the processes in (1) and (2).

For $A=\mathrm{H}$ only chemi-ionization processes with $n \geq 4$ and $\left(n-n^{\prime}\right)$-mixing processes with $\min \left(n, n^{\prime}\right) \geq 4$ can be described on the basis of the DRM, which caused by the existence of the stable negative ion $\mathrm{H}^{-}$. In contrast, for $A=\mathrm{He}$, the excited helium state with $n=3$ can be taken as the lower boundary of the block of Rydberg states. This was also used in Mihajlov et al. (2003), who focused on the chemi-ionization processes in DB white dwarf atmospheres.

The processes (1) and (2) are characterized by the excitation and deexcitation rate coefficients $K_{n ; n+p}(T)$ and $K_{n ; n-p}(T)$, where $T$ is the local temperature of the atomic particles in the considered stellar atmosphere. In accordance with the aim of this
Table 1. Excitation rate coefficients $K_{3 ; 3+p}(T)$ for $1 \leq p \leq 5$ in $\left[10^{-9} \mathrm{~cm}^{3} \mathrm{~s}^{-1}\right]$.

\begin{tabular}{cccccccc}
\hline \hline & \multicolumn{7}{c}{$T$} \\
& \multicolumn{7}{c}{$\left[10^{3} \mathrm{~K}\right]$} \\
\cline { 2 - 8 }$p$ & 5 & 6 & 8 & 10 & 12 & 16 & 20 \\
\hline 1 & 1.418 & 1.723 & 2.187 & 2.514 & 2.753 & 3.074 & 3.277 \\
2 & 0.197 & 0.271 & 0.401 & 0.504 & 0.585 & 0.701 & 0.778 \\
3 & 0.062 & 0.091 & 0.146 & 0.192 & 0.230 & 0.286 & 0.324 \\
4 & 0.028 & 0.042 & 0.071 & 0.096 & 0.117 & 0.148 & 0.170 \\
5 & 0.015 & 0.023 & 0.040 & 0.055 & 0.068 & 0.088 & 0.102 \\
\hline
\end{tabular}

work, these rate coefficients are defined as the quantities that determine the mean rates of transition (per single excited atom) caused by the processes (1) and (2) between whole shells with a given $n$ and $n \pm p$. They are determined here using a method similar to that described in Mihajlov et al. (2008): the excitation rate coefficients $K_{n ; n+p}(T)$ are calculated directly and numerically in the semi-classical way, while the deexcitation rate coefficients $K_{n ; n-p}(T)$ are determined later according to the principle of thermodynamical balance. All necessary expressions are given in Mihajlov et al. (2008).

For the DB white dwarf atmospheres considered here, the values of $K_{n ; n+p}(T)$ and $K_{n ; n-p}(T)$, which are used in Eqs. (1) and (2) for all necessary temperatures, can be calculated in principle by fitting the values of $K_{n ; n+p}(T)$ presented in Table 2 of Mihajlov et al. (2008), but only for the region $4 \leq n \leq 8$. Bearing this in mind, Table 1 shows the values of the excitation rate coefficients $K_{3 ; n+p}(T)$ when $1 \leq p \leq 5$. These values, together with Table 1 in Mihajlov et al. (2008), enable the calculation of $K_{n ; n+p}(T)$ and $K_{n ; n-p}(T)$ for the entire block, $3 \leq n \leq 8$.

The concurrent electron-Rydberg atom processes (3) are characterized here by the rate coefficients $\alpha_{n ; n \pm p}\left(T_{\mathrm{e}}=T\right)$, where $T_{\mathrm{e}}$ denotes the electron temperature, which in the general case is not equal to the atomic temperature. In the current study, the values of $\alpha_{n ; n \pm p}(T)$ are determined from the corresponding expressions given in Vriens \& Smeets (1980) and Johnson (1972). We note that ion-atom non elastic collisional processes, although they are also characterized by long-range interaction, cannot be concurrent. The reason is the huge difference in masses of electron and ion, which causes the impact ion-atom velocity to be several orders of magnitudes lower than electron-atom impact velocity.

To estimate the relative efficiency of processes (1) and (2) and the concurrent processes (3), it is sufficient to study the behavior of quantities $F_{n}^{(+)}$and $F_{n}^{(-)}$in the considered DB white dwarf atmospheres, which are given by the expressions

$F_{n}^{(+)}=\frac{\sum_{p=1}^{5} K_{n, n+p} \cdot N(n) \cdot N(1)}{\sum_{p=1}^{5} \alpha_{n, n+p} \cdot N(n) \cdot N_{\mathrm{e}}}=\frac{\sum_{p=1}^{5} K_{n, n+p}}{\sum_{p=1}^{5} \alpha_{n, n+p}} \cdot \eta, \quad n \geq 3$,

and

$F_{n}^{(-)}=\frac{\sum_{p=1}^{n-3} K_{n, n-p} \cdot N(n) \cdot N(1)}{\sum_{p=1}^{n-3} \alpha_{n, n-p} \cdot N(n) \cdot N_{\mathrm{e}}}=\frac{\sum_{p=1}^{n-3} K_{n, n-p}}{\sum_{p=1}^{n-3} \alpha_{n, n-p}} \cdot \eta, \quad n \geq 4$,

where $N(n), N(1)$, and $N_{\mathrm{e}}$ are the local densities of all Rydberg helium atoms in the states with a given $n \geq 3$, of a helium atom in the ground state, and of free electrons, respectively; and the products $K_{n, n \pm p} \cdot N(n) \cdot N(1)$ and $\alpha_{n, n \pm p} \cdot N(n) \cdot N_{\mathrm{e}}$ are the partial atom- and electron-Rydberg atom excitation/deexcitation fluxes. The factor $\eta$ is defined here by the relation

$\eta \equiv N(1) / N_{\mathrm{e}} \cong(\text { ionization degree })^{-1}$, 


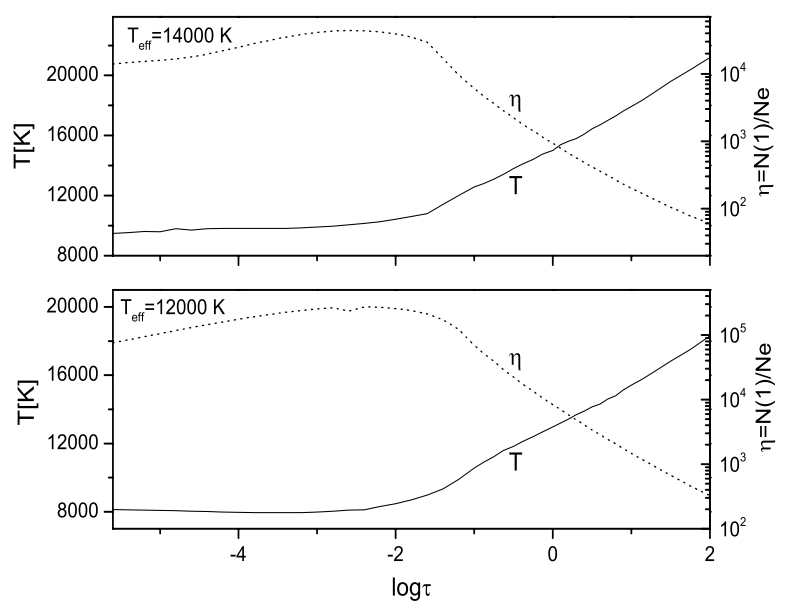

Fig. 1. Parameters (temperature $T$ - full line and $\eta=N(1) / N_{\mathrm{e}}-$ dotted) for DB white dwarf atmosphere models with $\log g=8$ and $T_{\text {eff }}=12000 \mathrm{~K}$ and $14000 \mathrm{~K}$ from Koester (1980) as functions of $\log \tau$, where $\tau$ is the Rosseland optical depth.
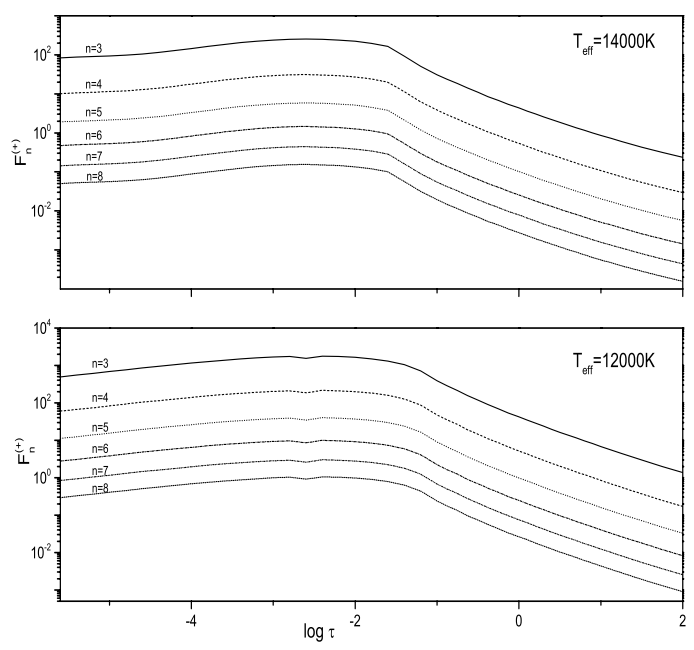

Fig. 2. Behavior of quantities $F_{n}^{(+)}$, given by Eq. (4) with $3 \leq n \leq 8$, on the basis of DB white dwarf atmosphere models from Koester (1980) for $\log g=8$ and $T_{\text {eff }}=12000 \mathrm{~K}$ and $14000 \mathrm{~K}$, as functions of $\log \tau$, where $\tau$ is the Rosseland optical depth.

and its behavior in the atmospheres of the considered DB white dwarfs (which is very important for the studied processes) is presented in Fig. 1.

\section{Results and discussion}

Figure 2 illustrates the behavior of the quantity $F_{n}^{(+)}$with $3 \leq n \leq$ 8 in the DB white dwarf atmospheres described by the models presented in Koester (1980), for $\log g=8$ and $T_{\text {eff }}=12000 \mathrm{~K}$ and $14000 \mathrm{~K}$. All results were obtained using the data from Koester (1980), and these quantities are presented as functions of $\log \tau$, where $\tau$ is the Rosseland optical depth. Figure 2 unambiguously shows that for DB white dwarf atmospheres with $T_{\text {eff }} \lesssim 14000 \mathrm{~K}$, the excitation processes (1) in the lower part of the Rydberg block ( $3 \leq n \leq 8)$ must be taken into account as a new important factor that influences the helium Rydberg-state populations. Figure 2 shows that in the parts of the atmosphere where the ionization degree is $\lesssim 10^{-3}$, the processes (1) with $n=3,4$, and 5 are dominant with respect to the known concurrent processes (3); for $n=6$ and 7 they are dominant in the parts where the ionization degree is $\lesssim 10^{-4}$; and in the remaining parts their efficiency is comparable with the efficiency of the processes (3). Even the efficiency of the processes (1) with $n=8$ is close to, or at least comparable with, the efficiency of the processes (3) in the parts where the ionization degree is $<10^{-4}$.

These facts are especially important for the considered DB white dwarf atmospheres, because for any temperature from the relevant temperature regions, the corresponding Boltzman distribution of the excited helium atom populations will have a distinct minimum (known as the "bottleneck") only in the region $3 \leq n<8$. Thus, it is correct to expect that even a considerable perturbation of the mentioned distribution would not result in a significant change in the position of this minimum. Hence, it follows that the excitation processes (1) should by all means be taken into account in any future non-LTE model of DB white dwarf atmospheres with similar parameters.

Examining the behavior of the quantities $F_{n}^{(-)}$with $4 \leq n \leq 8$ in the same DB white dwarf atmospheres would lead to the same conclusions, including the necessity of introducing the deexcitation processes (2) into the mentioned models. For this reason, the efficiencies of the considered $\left(n-n^{\prime}\right)$-mixing processes are only illustrated in Fig. 2.

Finally, we emphasize that these results are relevant not only to the atmospheres of the DB white dwarfs mentioned here, but also to a considerably wider range of helium-rich stellar atmospheres. For instance, an entire array of white dwarfs of other types, as described in Wegner \& Koester (1985), Dufour et al. (2006, 2007), have similar parameters. Consequently, processes (1) and (2) must be just as important as the concurrent processes (3). Overall, the findings suggest that the considered atom-Rydberg atom $\left(n-n^{\prime}\right)$-mixing processes (1) and (2) should be included $\mathrm{ab}$ initio in the modeling of the helium-rich white dwarf atmospheres.

Acknowledgements. The authors are grateful to the Ministry of Education, Science and Technological Development of the Republic of Serbia for the support of this work within the projects 176002 , III 4402 .

\section{References}

Barklem, P. S. 2007, A\&A, 466, 327

Dufour, P., Bergeron, P., Schmidt, G. D., et al. 2006, ApJ, 651, 1112

Dufour, P., Bergeron, P., Liebert, J., et al. 2007, ApJ, 663, 1291

Ignjatović, L. M., Mihajlov, A. A., Sakan, N. M., Dimitrijević, M. S., \& Metropoulos, A. 2009, MNRAS, 396, 2201

Janev, R. K., \& Mihajlov, A. A. 1979, Phys. Rev. A, 20, 1890

Janev, R. K., \& Mihajlov, A. A. 1980, Phys. Rev. A, 21, 819

Johnson, L. C. 1972, ApJ, 174, 227

Koester, D. 1980, A\&AS, 39, 401

Mashonkina, L. 2009, Phys. Scr. T, 134, 014004

Mihajlov, A. A. 1982, in Proceedings of international conference on plasma physics. G

Mihajlov, A. A., Dimitrijević, M. S., \& Djurić, Z. 1996, Phys. Scri., 53, 159

Mihajlov, A. A., Ignjatović, L. M., Vasilijević, M. M., \& Dimitrijević, M. S. 1997, A\&A, 324, 1206

Mihajlov, A. A., Jevremović, D., Hauschildt, P., et al. 2003, A\&A, 403, 787

Mihajlov, A. A., Ignjatović, L. M., Djurić, Z., \& Ljepojević, N. N. 2004, J. Phys. B At. Mol. Phys., 37, 4493

Mihajlov, A. A., Ignjatović, L. M., \& Dimitrijević, M. S. 2005, A\&A, 437, 1023

Mihajlov, A. A., Jevremović, D., Hauschildt, P., et al. 2007, A\&A, 471, 671

Mihajlov, A. A., Ignjatović, L. M., Srećković, V. A., \& Djurić, Z. 2008, J. Quant. Spec. Radiat. Transf., 109, 853

Mihajlov, A. A., Ignjatović, L. M., Srećković, V. A., \& Dimitrijević, M. S. 2011, ApJS, 193, 2

Mihajlov, A. A., Srećković, V. A., Ignjatović, L. M., \& Klyucharev, A. N. 2012, J. Cluster Sci., 23, 47

Vernazza, J. E., Avrett, E. H., \& Loeser, R. 1981, ApJS, 45, 635

Vriens, L., \& Smeets, A. H. M. 1980, Phys. Rev. A, 22, 940

Wegner, G., \& Koester, D. 1985, ApJ, 288, 746 